\title{
Dual iris authentication system using dezert smarandache theory
}

\author{
Kamel Ghanem Ghalem ${ }^{1}$, Fatiha Hendel ${ }^{2}$ \\ ${ }^{1}$ Ecole Supérieure En Génie Electrique et Energétique, Algérie \\ ${ }^{2}$ Université des Sciences et de la Technologie d'Oran Mohamed Boudiaf, Algérie
}

\section{Article Info \\ Article history: \\ Received Nov 25, 2018 \\ Revised May 16, 2019 \\ Accepted Jun 26, 2019}

\section{Keywords:}

Authentication

Biometric

Dezert Smarandache theory Iris

\begin{abstract}
In this paper, a dual iris authentication using Dezert Smarandache theory is presented. The proposed method consists of three main steps: In the first one, the iris images are segmented in order to extract only half iris disc that contains relevant information and is less affected by noise. For that, a Hough transform is used. The segmented images are normalized by Daugman rubber sheet model. In the second step, the normalized images are analyzed by a bench of two 1D Log-Gabor filters to extract the texture characteristics. The encoding is realized with a phase of quantization developed by $\mathrm{J}$. Daugman to generate the binary iris template. For the authentication and the similarity measurement between both binary irises templates, the hamming distances are used with a previously calculated threshold. The score fusion is applied using DSmC combination rule. The proposed method has been tested on a subset of iris database CASIA-IrisV3-Interval. The obtained results give a satisfactory performance with accuracy of $99.96 \%$, FAR of $0 \%$, FRR of $3.89 \%$, EER of $2 \%$ and processing time for one iris image of $12.36 \mathrm{~s}$.
\end{abstract}

Copyright (C) 2019 Institute of Advanced Engineering and Science. All rights reserved.

\section{Corresponding Author:}

Kamel Ghanem Ghalem,

Ecole Supérieure En Génie Electrique et Energétique,

BP 64 CH2 ACHABA HANIFI USTO 31000 Oran, Algérie.

Email: ghalem_kamel_ghanem@esgee-oran.dz

\section{INTRODUCTION}

When individuals log onto computers, or access an ATM, or pass through airport security, they have to reveal their identities. For this, individuals use passwords, ATM cards, and passeports to prove their identities. However, passwords can be forgotten, and ATM cards or passports can be lost or stolen. In contrary, the biometric modalities (Fingerprint, face, iris, ...etc) speak to what and they also allow to prove our identity.

However, the unimodal biometric systems using one biometric modality for recognition cannot guarantee at present an excellent recognition rate. Furthermore, these systems suffer from limitations such as sensitivity to noise, data quality, non-universality, and spoof attacks. To overcome these problems, Multimodal biometric systems, which combine multiple biometric modalities, have been developed on purpose to achieve a better recognition rate.

The popular fusion method of the biometric traits can be done at tow stages of recognition system:

1. Fusion at feature extraction level

The data is acquired from each sensor is utilized to generate a feature vector. Then, the features are fused to form one feature vector.

2. Fusion at matching score level

The matching score of each system is combined and compared with the stored template. 
We use as modality for recognition of individuals: iris, since their texture is

- Stable throughout the life of a person, unlike the fingerprint.

- Unique for each person, unlike a facial feature in identical twins.

- Unfalsifiable contrary to the characteristics of the voice.

- Iris is an internal organ well protected from the external environment, but nevertheless measurable, in a rather little invasive way, by a simple image acquisition.

Daugman's algorithm [1] is one of the best iris algorithm known in biometrics. The algorithm consists to segment iris using Integro-Differential Operator and iris normalization is implemented using Daugman's polar representation. Then, iris encoding is applied using 2D Gabor filters to extract a binary code of 256 bytes. The Matching is processed by computing similarity between two iris codes using Hamming distance. The more Hamming distance is small, the more both codes are similar. A distance of 0 corresponds to a perfect match between both iris images, while two iris images of different person will have a Hamming distance close to 0.50 .

In 1997, Wildes [2] proposed a novel iris recognition system compared to Daugman algorithm [1]. The acquisition of iris is done by a CCD Camera in low luminosity. Then, the iris is segmented using Circular and Elliptic Hough transform and is normalized using a transformation function of pixels. After that, the iris is filtered by Laplacian of Gaussian filters with four different resolution levels. A normalized correlation is calculated for every resolution levels. The median of the values of correlations is computed for the filtered image. The fusion of four values is applied using Fisher's linear discriminant.

In 1998, W. Boles and B. Boashash [3], presented a new algorithm for recognition of individuals from iris images. The algorithm is insensitive to variation in the lighting conditions and noise levels. A Median filter is used for preprocessing. The advantage of this technique is to extract a features vector from 1D signals rather than 2D images analyzed in [1], [2] using zero-crossings of the dyadic wavelet transform at various resolution levels. Only a few selected intermediate resolutions are used for matching. The matching is applied using different dissimilarity functions. Thus, make the algorithm faster and less sensitive to noise and quantification error.

In 2004, Ma et al. [4] presented an efficient algorithm for iris recognition. The iris is segmented by Canny filter and Hough transform. Then, the iris is normalized by histogram equalization. After that, A 1D Wavelet Transform is used to represent resulting 1D intensity signals. The position of local sharp variation points is registered as features. The matching is effectuated using the similarity function (exclusive or operation). This algorithm is efficient and faster than Daugman's algorithm [1].

In [5], the researchers proposed a modified Masek approach and a comparative study of the performance of the following methods: radial segmentation, Masek segmentation approach, modified Masek approach. The proposed method tested on Casia Iris Database V3 showed a good performance in terms of accuracy and processing time.

R. Biswas [6] has introduced an iris recognition system that includes different steps: segmentation, normalization, feature extraction, and classification. The segmentation of the pupil is performed by the Hough transform. Experimental results showed a recognition rate of $92 \%$.

In [7], the authors proposed an iris recognition system based on "Fractal dimension of box-counting method". First, the iris is segmented by Hough transform and is normalized by Daugman's rubber sheet model. Then, the feature extraction is processed by box counting. Finally, the matching is established using K-nearest Neighbor and Euclidean distance. Experiments tested on Casia Interval V4 database showed a good recognition rate equal to $92.63 \%$.

D. Bobeldyk and A. Ross [8] have developed a method for predicting eye color from NIR iris images. Researchers have shown that a texture based approach based on the BSIF is more efficient than the intensity based approach based on raw pixel values. Experiments tested on the BioCOP database showed a good recognition rate of $90 \%$. The BSIF distinguished "light color iris" and "dark color irides" using the SVM classifier.

The authors [9] presented a new method of classifying faked iris images of different patterns such as printed irises, contact lenses. The new classification method learns different characteristics of faked iris images by CNN and identifies legitimate and faked iris images using "Hierarchical Multi-Class". The tests carried out on the different databases: ND-contact, Casia-Iris-Interval and Casia-Iris-Syn, LivDet-Iris-2017Warsaw showed a recognition rate equal to $100 \%$ and $\mathrm{FAR}=0 \%, \mathrm{FRR}=0 \%$.

H. G. Daway et al. [10] presented a new method for detecting the pupil. The method involves several steps, the most important of which depends on the difference in color and intensity between the pupil and its neighborhood. These characteristics are very important to locate and extract the pupil. Thus, the pupil is a region of very high intensity (color) compared to its neighborhood. The experimental results showed a recognition rate equal to $100 \%$.

Int J Elec \& Comp Eng, Vol. 9, No. 6, December 2019：4703 - 4712 
In purpose to improve an overall performance in terms of recognition rate and mitigate errors, the researchers have used more than one biometric trait, and thus, the multibiometric systems have emerged. Numerous multi-biometric systems have been developed, which fusion is made at Matching score.

In [11], the researchers proposed a new approach for recognition using both irises. The iris is segmented using the Canny filter and Hough transform, then the segmented iris is normalized by J. Daugman's rubber sheet model. The iris feature extraction is carried out using convolution of the normalized iris with 1D Log-Gabor filters then the phase of filtered iris is quantized in order to generate a binary code. The Hamming distance is used for Matching. The Matching operation consists in comparing the two iris feature vectors of a person with the others; if the Hamming Distances are less than the threshold then the person is identified. The experimental results showed a good recognition rate equal to $99.92 \%$ with an FRR $=9.96 \%$, while for unimodal systems (left iris and right iris) the recognition rate is equal to $99.87 \%$ with an FRR $=14.62 \%$ and $\mathrm{FPR}=15.68 \%$.

In [12], the authors presented the framework for multimodal biometric fusion based on the uncertainty concept of Dempster-Shafer theory. A combination of quality measures and the accuracy of classifiers (equal error rate) are proposed to encode the uncertainty concept to improve the fusion. The proposed method revealed a good performance with an EER equal to $1 \%$.

R. Dwivedi and S. Dey [13] proposed a cancelable multibiometric system using score level fusion. The fusion of scores was applied by MC weighting at first level and RA weighting at the second level. The comparative analysis shows that the proposed fusion method outperforms the existing weighting approaches.

In this works, we extract only the interior half of the iris disc rather than the whole iris disc, which contains the most relevant information and it is less affected by noise. In addition, we combined two sources of information (left iris and right iris) with high degree of conflict using Dezert Smarandache Theory, which solves the problem of highly redistributed masses conflicts arising under the Dempster shafer theory. The reminder paper is organized as follows: the research method is described in section 2, Results and Analysis are presented in section 3, conclusions are provided in section 4.

\section{RESEARCH METHOD}

The key idea of our work is to extract only the discriminant information from iris texture and proposes Dezert Smarandache Theory (DSmT) at score level fusion to operate under uncertainty in goal to achieve a good performance. The proposed method is composed of four main stages: preprocessing, feature extraction, fusion, and matching.

\subsection{Preprocessing stage}

First, the iris images require going through the preprocessing phase including segmentation and normalization.

\subsubsection{Iris segmentation}

The segmentation of iris is realized by commonly Edge detector method: Hough transform.

- Hough transform

- Algorithm

- Generate edge map using the Canny filter.

- Canny parameters:

The standard deviation of Gaussian smoothing filter: $\sigma=2$.

Weighting for vertical gradients $=0$.

Weighting for horizontal gradients $=1$.

- Increase contrast in dark iris region.

Image gamma value: enhance the contrast of bright regions: $\gamma=1.9$.

- Detect pixel corresponding to the local maxima

Distance in pixel units to be looked at on each side of each pixel when determining, whether it is a local maximum or not: $\mathrm{d}=1.5$.

- Binarize iris image using Hysteresis thresholding.

Low threshold $\mathrm{T}_{1}=0.19$.

High threshold $\mathrm{T}_{2}=0.20$.

The Figure 1 illustrates a differents step of Hough transform. Then, a Circular HoughTransform detects at first the iris/sclera boundary and the iris/pupil boundary. The eyelashes are detected by global thresholding $(\mathrm{T}=100)$. 


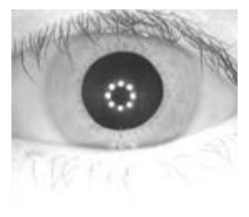

Iris image

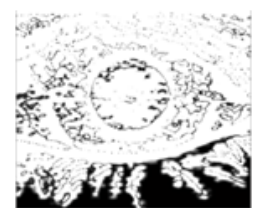

Edge map

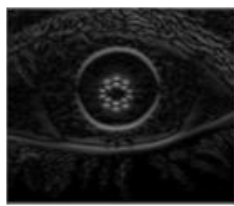

Increase contrast

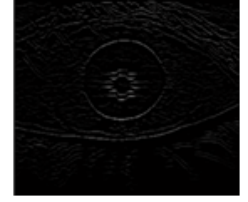

Non-maxima suppression

Figure 1. Differents step of Hough Transform.

In this work, the objective is to extract only relevant information from iris, which is represented by the structural variation of the iris texture (high gradient areas), only the internal half of the iris disc is exploited rather than whole, because it contains the most relevant information [14] and it is less affected by the noise as shown in Figure 2. Indeed, the proposed technique decreases the complexity and the computation load without losing information as shown in Table 1. From Table 1, we denote that treatment using only half iris disc is more efficient with an accuracy of $99.96 \%$ and processing time for one iris image of $12.36 \mathrm{~s}$ than the treatment using a whole iris disc with accuracy $99.87 \%$ of and processing time of $22.88 \mathrm{~s}$.

$$
\mathrm{r}_{\text {half of iris disc }}=\mathrm{r}_{\text {pupil }}+\left(\mathrm{r}_{\text {iris }}-\mathrm{r}_{\text {pupil }}\right) / 2
$$
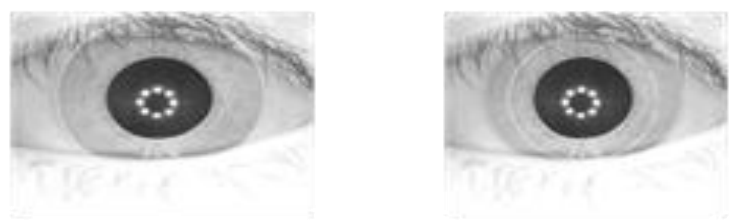

Figure 2. Delimitation of only the internal half of iris disc

Table 1. Comparison: Total iris disc vs Half iris disc

\begin{tabular}{ccc}
\hline & Accuracy $(\%)$ & Processing time for one iris image (s) \\
\hline Total iris disc & 99.87 & 22.88 \\
Half iris disc & 99.96 & 12.36 \\
\hline
\end{tabular}

\subsubsection{Iris normalization}

The iris disc does not always have the same dimension, even for eye images of the same person; this is due to various problems as follows:

a) Different acquisition conditions of the eye images. Dilation and contraction of the pupil due to the variation of the illumination level.

b) The circles of iris and pupil are not concentric.

In order to overcome these problems, a stage of normalization is applied. It consists of transforming the region of the iris disc to rectify the dimensions of all the iris discs, by using the homogenous rubber sheet model proposed by Daugman [1]. It transforms each point in the iris area to the polar coordinates $(\mathrm{r}, \theta)$, where $r$ is on the interval $[0,1]$ and $\theta$ is angle $[0,2 \pi]$, as illustrated in Figure 3.

In our system, $(20 * 240)$ points were used, but only $(10 * 240)$ points corresponding to the internal half of the iris disc that contains the most relevant information and which is less affected by noise, are retained for the next steps of the processing, as shown in Figure 4. 


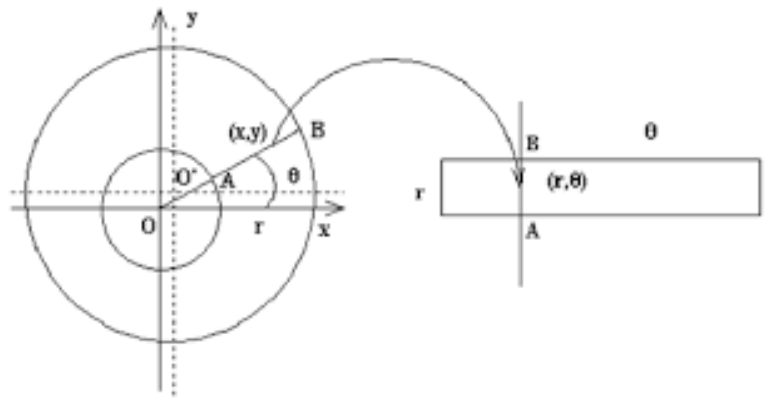

Figure 3. Daugman rubber sheet model [1]

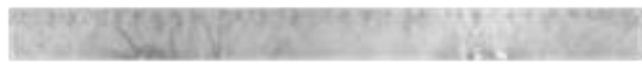

Figure 4. Normalization of the segmented iris

\subsection{Feature extraction stage}

After that, the feature extraction stage is applied in purpose to extract the most discrimination information present in the iris region. For this reason, a bench of two $1 \mathrm{D}$ Log-Gabor filter is used.

a) $1 \mathrm{D}$ log-gabor filter

- The Fast Fourier Transform is applied for each line of the normalized matrix image (FFT to 1D signals).

- Then, the Inverse Fast Fourier Transform IFFT is applied on the multiplication FFT (1D signals) by a 1D Log-Gabor Filter.

- The frequency response of a 1D Log-Gabor filter is given by:

$$
G(f)=\exp \left(-\frac{\log \left(\frac{f}{f_{0}}\right)^{2}}{\log \left(\frac{f}{f_{0}}\right)^{2}}\right)
$$

\section{b) Parameters setting}

- A bench of two 1D Log-Gabor filters is used.

- The standard deviation of the 1D Log-Gabor wavelet is given by $\sigma=2$.

- The center frequency of the 1D Log-Gabor wavelet is given by $f_{0}=0.05$

Indeed, the phase of a filtered image was quantized using four-quadrants of Daugman [1], when going from one quadrant to an adjacent quadrant, one bit is changed as shown in Figure 5. The encoding process produces a bitwise template containing a number of information bits as shown in Figure 6, the total number of bits in the template (9600 bits) will be the angular resolution (240) times the radial resolution (10), times 2 , times the number of filters used (2).

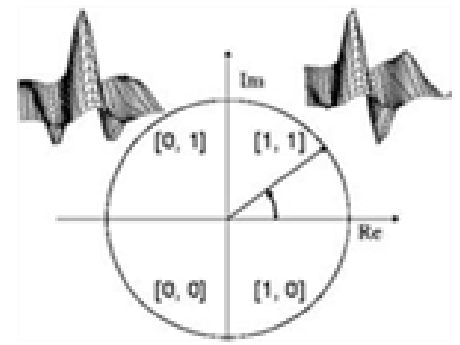

Figure 5. Quantization phase [1]

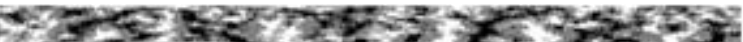

Figure 6. Iris code 


\subsection{Matching stage [Comment : Matching score come before Fusion stage ]}

The matching consists in comparing two iris code using Hamming distance. The Hamming Distance (HD) is defined by:

$$
\mathrm{HD}=\sum_{\mathrm{j}=1}^{\mathrm{N}} \mathrm{X}_{\mathrm{j}} \oplus \mathrm{Y}_{\mathrm{j}}
$$

Where $X_{j}$ and $Y_{j}$ are the two bitwise iris code, $N$ is the number of bits in each iris code. Literally, the Hamming distance calculates the number of different and valid bits for the two iris code between $X_{j}$ and $Y_{j}$. The number of translation bits that compensates the rotation of the iris needs to be fixed. We applied a translation of the iris code in an interval $[-\mathbf{3},+\mathbf{3}]$ bits. We take into consideration the minimum Hamming distance.

\subsection{Fusion stage}

In this stage, score level fusion using Dezert Smarandache theory (DSmT) was applied on goal to improve the performance of the dual iris system.

Score level fusion

Matching score level fusion combines the scores generated by multiple classifiers relating to the left and right iris to affirm the veracity of the claimed identity. The Dezert Smarandache theory operates under hyperpower set $\mathrm{D}^{\Theta}$. Thus, DSmT is able to function properly not only with the unions but also with intersections. Dsm Classic has combination rule [15-17]:

$$
\mathrm{m}(\mathrm{C})=\sum_{\mathrm{A} \cap \mathrm{B}=\mathrm{C}} \mathrm{m}_{1}(\mathrm{~A}) \mathrm{m}_{2}(\mathrm{~B}) ; \mathrm{A}, \mathrm{B} \in \mathrm{D}^{\Theta}, \forall \mathrm{C} \in \mathrm{D}^{\Theta}
$$

Example

$$
\begin{aligned}
& \Theta=\left\{\mathrm{S}_{\text {left }}, \mathrm{S}_{\text {right }}\right\} \\
& \mathrm{D}^{\Theta}=\left\{\varnothing, \mathrm{S}_{\text {left }}, \mathrm{S}_{\text {right }}, \mathrm{S}_{\text {left }} \cup \mathrm{S}_{\text {right }}, \mathrm{S}_{\text {left }} \cap \mathrm{S}_{\text {right }}\right\}
\end{aligned}
$$

$\emptyset$ : Empty set

$\mathrm{S}_{\mathrm{left}}$ : Hypothesis assuming that two individuals have same left iris.

$\mathrm{S}_{\text {Right }}$ : Hypothesis assuming that two individuals have a same right iris.

$\mathrm{S}_{\text {left }} \cup \mathrm{S}_{\text {right }}:$ Hypothesis assuming that two individuals have different iris

$\mathrm{S}_{\text {left }} \cap \mathrm{S}_{\text {right }}$ : Hypothesis assuming that two individuals have different iris.

\subsection{Decision}

The decision is made by fixing a threshold. The two irises compared will be considered as belonging to the same person if the calculated score is inferior to a threshold.

\section{RESULTS AND ANALYSIS}

\subsection{Simulation environment}

The proposed method has been tested on a subset of iris database CASIA-IrisV3-Interval [18] in order to evaluate its performance in authentication mode. The subset contains 1180 eye images of 118 individuals (classes), and each individual has five iris samples for the left eye and five iris samples for the right eye.

\subsection{Performance metrics}

False Reject Rate (FRR): also known as Type I error, is the measure of the probability that the biometric security system will incorrectly reject an access attempt by an authorized user.

- False Accept Rate (FAR): also known as Type II error, is the measure of the probability that the biometric security system will incorrectly accept an access attempt by an unauthorized user.

- $\quad$ EER (Equal Error Rate): The EER is the operating point for which the False Reject Rate (FRR) is equal to the False Accept Rate (FPR). 


\subsection{Decidability}

Decidability [1] is the best metric which indeed takes into account the mean and standard deviation of the intra-class and inter-class distributions:

$$
\mathrm{d}^{\prime}=\frac{\left|\mu_{\mathrm{s}}-\mu_{\mathrm{d}}\right|}{\sqrt{\frac{\left(\sigma_{\mathrm{s}}^{2}+\sigma_{\mathrm{d}}^{2}\right)}{2}}}
$$

Decidability $\mathrm{d}^{\prime}$ is a distance in standard deviations calculated using (7), which is a function of the magnitude of the difference between the mean of the intra-class distribution $\mu_{s}$, and the mean of the interclass distribution $\mu_{\mathrm{d}}$, the standard deviation of the intra-class and inter-class distributions, $\sigma_{\mathrm{s}}$ and $\sigma_{\mathrm{d}}$ respectively.

Table 2.Discidability table for various numbers of bit-shifts

\begin{tabular}{cccccc}
\hline Number of shifts & $\mu_{\mathrm{s}}$ & $\sigma_{\mathrm{s}}$ & $\mu_{\mathrm{d}}$ & $\sigma_{\mathrm{d}}$ & $\mathrm{d}^{\prime}$ \\
\hline 0 & 0.3300 & 0.0723 & 0.4914 & 0.0284 & 3.4314 \\
1 & 0.3137 & 0.0697 & 0.4860 & 0.0279 & 3.8149 \\
2 & 0.3072 & 0.0668 & 0.4812 & 0.0269 & 4.0264 \\
$\mathbf{3}$ & $\mathbf{0 . 3 0 4 4}$ & $\mathbf{0 . 0 6 5 3}$ & $\mathbf{0 . 4 7 7 2}$ & $\mathbf{0 . 0 2 5 8}$ & $\mathbf{4 . 0 7 4 2}$ \\
4 & 0.3032 & 0.0646 & 0.4738 & 0.0247 & 4.0431 \\
5 & 0.3028 & 0.0642 & 0.4709 & 0.0238 & 3.9907 \\
6 & 0.3025 & 0.0639 & 0.4684 & 0.0230 & 3.9362 \\
7 & 0.0637 & 0.0642 & 0.0223 & 0.0216 & 3.8862 \\
8 & 0.3023 & 0.0635 & 0.4645 & 0.0216 & 3.8303 \\
9 & 0.3022 & 0.0634 & 0.4629 & 0.0211 & 3.7999 \\
10 & 0.2758 & 0.0639 & 0.4643 & 0.0201 & 4.3960 \\
\hline
\end{tabular}

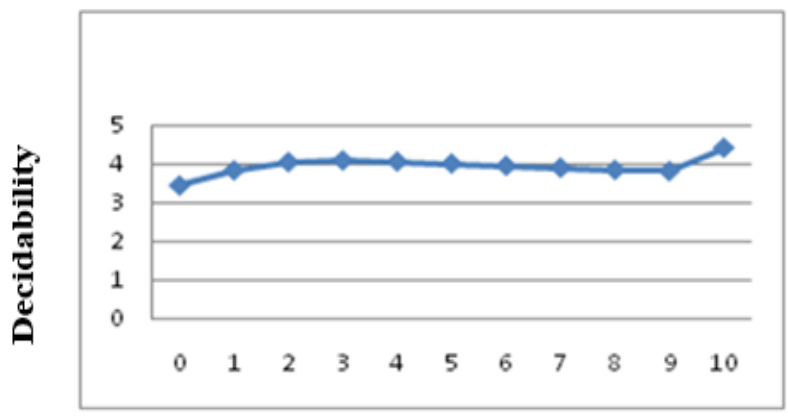

Number of bits shifted in left and right

Figure 7. Decidability curve for various numbers of bit-shifts

Using (7), several different decidability are found out using 0-bit shift to 10-bit shift towards both left and right iris templates. The higher decidability is equal to 4.0742 at 3 bit shift as shown in Table 2 and Figure 7 that guarantees good separation of intra-class and inter-class distributions, which allows for more accurate recognition

\subsection{Score level fusion}

In fact, we calculated the fusion score $S_{f}$ using Haming distances obtained by comparing the individuals from their iris

$\mathrm{HD}_{\mathrm{L}}$ : Hamming distance obtained by comparing the individuals from their left iris.

$\mathrm{S}_{\mathrm{L}}: \quad$ Score obtained by comparing the individuals from their left iris.

$\mathrm{HD}_{\mathrm{R}}$ : Hamming distance obtained by comparing the individuals from their right iris.

$\mathrm{S}_{\mathrm{R}}$ : $\quad$ Score obtained by comparing the individuals from their right iris 


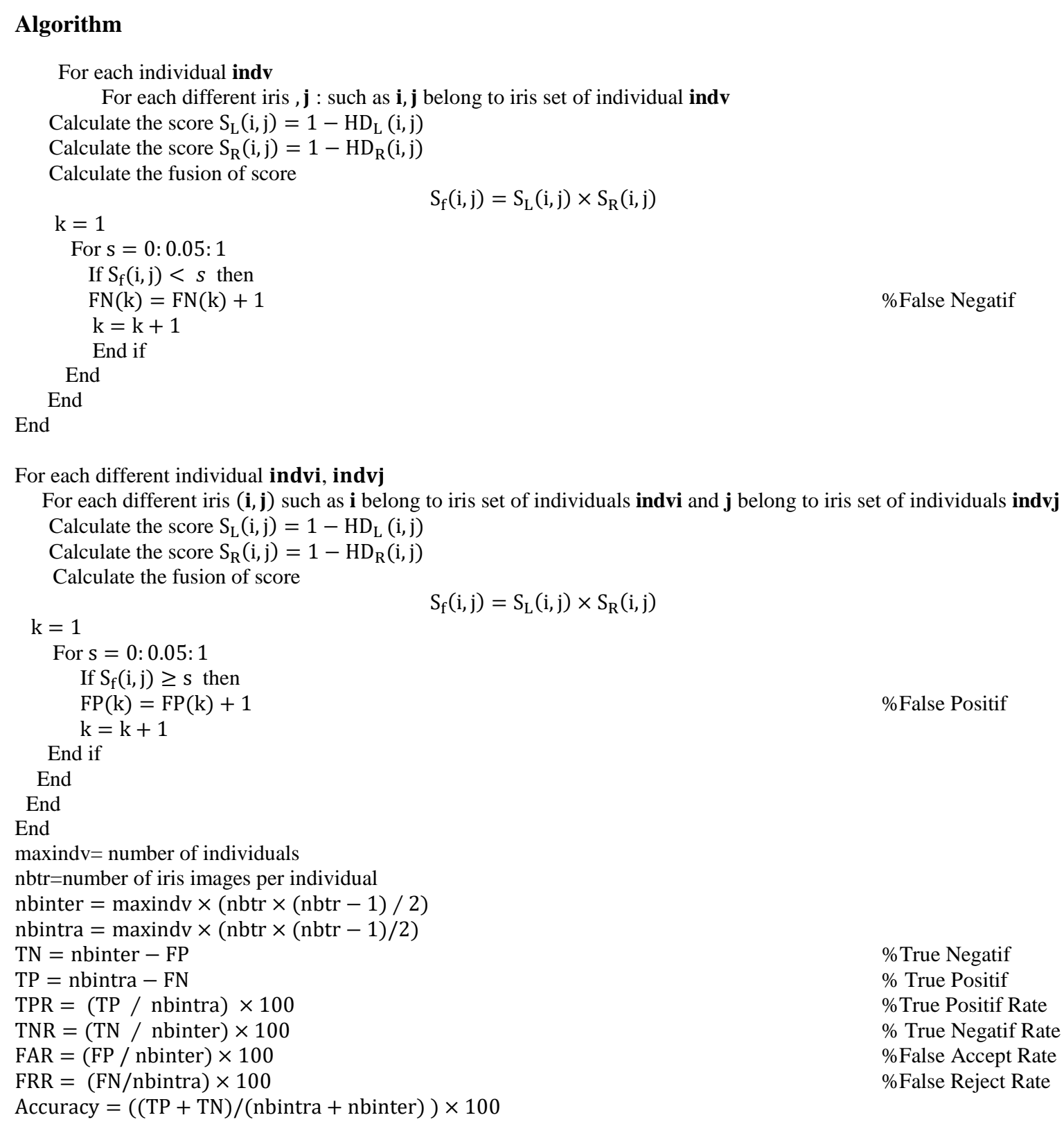

The dual iris system using DsmC at score level fusion reaches an accuracy rate of $99.96 \%$ and FAR of $0 \%$, FRR of $3.89 \%$, EER of $2 \%$ as shown in Figure 8 and Figure 9. We conclude from Figure 10 that the ROC (Receiver Operating Characteristic) of dual iris system using DsmC at score level fusion fit the origin, which proves the performance of our method.

\subsection{Comparison of various approaches}

We denote from Table 3, that the proposed dual iris authentication system gives a competitive performance with accuracy of $99.96 \%$, FAR of $0 \%$, FRR of $3.89 \%$, EER of $2 \%$ in comparison with other approchs. The problem of Dempster Shafer theory used in DST approach [12] (EER of 1\%) that consists of combining two sources of information with high degree of conflict is resolved. The proposed method is based on Dezert Smarandache Classic rule (DsmC) that solves this problem. Iftakhar and al [11] used the fusion method based on the AND rule that gives an accuaracy of $99.92 \%$, FAR of $0 \%$, FRR of $9.96 \%$, which is more drastic and leads to improve the FAR. R. Dwindi and S.Dey obtained less performance in term of accuracy equal to $98.89 \%$, EER of $0.69 \%$, which used a score fusion methods likes: MC weighting and RA weighting. These two methods using for optimization gives a little improvement to the performance of system. 


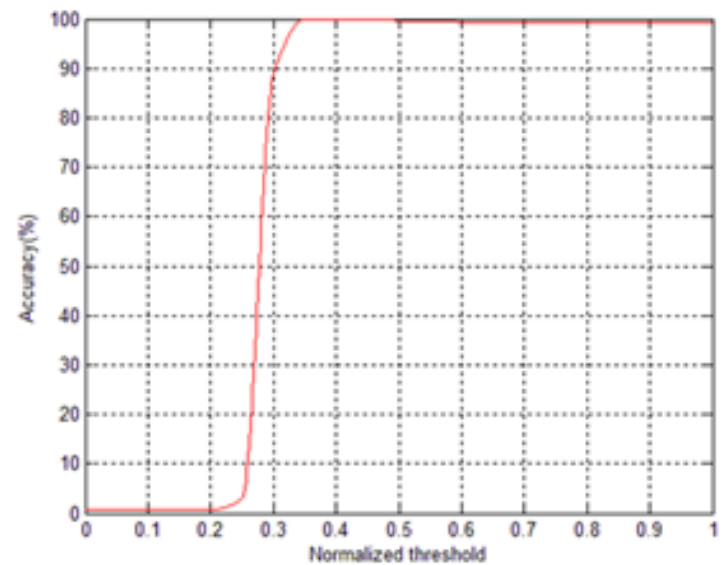

Figure 8 . The accuracy of the dual iris system

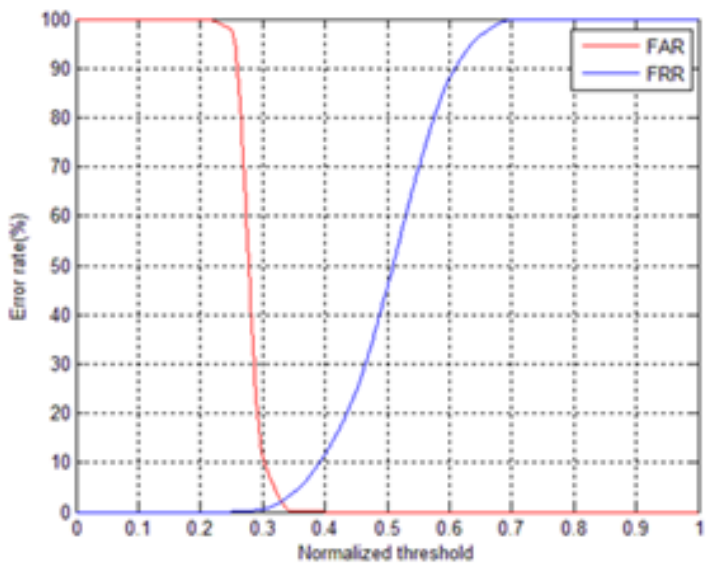

Figure 9. FRR and FAR of the dual iris system

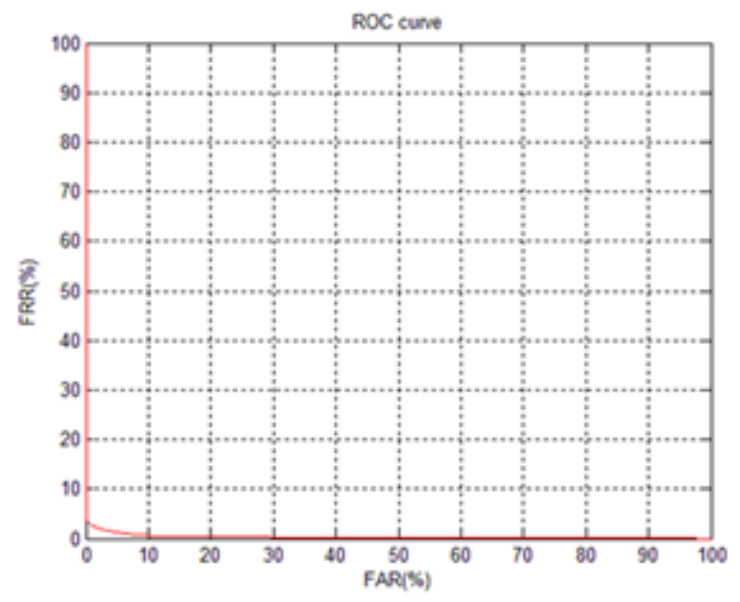

Figure 10. ROC of the dual iris system

Table 3. Accuracy and error for vaious approaches

\begin{tabular}{lcccc}
\hline \multicolumn{1}{c}{ Iris recognition systems } & Accuracy (\%) & FAR (\%) & FRR (\%) & EER (\%) \\
\hline Iftakhar and Ashraful Approach [11] & 99.92 & 0.00 & 9.96 & - \\
DST approach [12] & - & - & - & 1 \\
R. Dwivedi and S. Dey [13] & 98.89 & - & - & 0.69 \\
Proposal dual iris authentication system & 99.96 & 0.00 & 3.89 & 2 \\
\hline
\end{tabular}

\section{CONCLUSION}

The purpose of this work was to find out a dual iris authentication system that guarantees a good performance and to make sure that there is no false acceptance rate, which promises useful security applications. The proposed method consists in segmenting, to normalizing, characterizing and encoding the iris. For the segmentation part, the detection of the iris/pupil circles was performed by Hough circular transform. Only the interior half of the iris disc containing the most relevant information and less affected by noise, which reduces time complexity was extracted. Iris normalization part was performed by the Daugman rubber sheet model with a resolution of $10 \times 240$. This stage was analyzed by the bench of two 1 D Log-Gabor filters to extract the texture characteristics and the encoding was realized with a phase of quantization developed by J. Daugman to generate the binary iris template. For the authentication and the similarity measurement between both binary irises templates, the hamming distances are used with a previously calculated threshold. The score fusion is applied using Dezert Smarandache Classic (DSmC) rule. The experiment tested on Casia-iris v3-interval shows that the proposed system gives a good performance compared to others approaches with an accuracy of $99.96 \%$, FAR of $0 \%$, FRR of $3.89 \%$, EER of $2 \%$ and processing time for one iris image of $12.37 \mathrm{~s}$. 


\section{REFERENCES}

[1] Daugman J., "How iris recognition works," IEEE Trans.Circuits Syst. Video Techn, 14: 21-30, 2004.

[2] Wildes R. P et al. A machine-vision system for iris recognition. Machine Vision and Applications. 1996; 9: 1-8.

[3] Boles and Boashash B. A human identification technique using images of the iris and wavelet transform. IEEE Transactions on Signal Processing. 1998; 46: 1185-1188.

[4] Ma L et al. "Efficient iris recognition by characterizing key local variations," IEEE Transactions on Image Processing, 13: 739-750, 2004.

[5] Aydi W, Masmoudi N, Kamoun L. "A Fast and Accurate Circular Segmentation Method for Iris Recognition Systems," International Review on Computers and Software (IRECOS), 9 (3): 468-477, 2014.

[6] Biswas R, Uddin J, Hasan Md. J. "A New Approach of Iris Detection and Recognition," International Journal of Electrical and Computer Engineering (IJECE), 7(5): 2530-2536, 2017.

[7] Khotimah C and Juniati D. "Iris Recognition Using Feature Extraction of Box Counting Fractal Dimension," J. Physics: Conf.Series, 2018.

[8] Bobeldyk D and Ross A. "Predicting Eye Color from Near Infrared Iris Images," International Conference on Biometrics, 104-110, 2018.

[9] Yan Z, He L, Zhang M, Sun Z and. Tan T. "Hierarcical Multiclass Iris Classification for Liveness detection," International Conference on Biometrics, 47-53, 2018.

[10] Daway H. G, kareem H. H, Hashim A. R, "Pupil Detection Based on Color Difference and Circular Hough Transform," International Journal of Electrical and Computer Engineering (IJECE), 8(5): 3278-3284, 2018.

[11] Iftakhar Hasan K.M, Ashraful Amin M. "Dual iris matching for biometric identification," Signal Image and Video Processing. 8(8): 1605-1611, 2014.

[12] Nguyen K, Denman S. "Score-Level Multibiometric Fusion Based on Dempster-Shafer Theory Incorporating Uncertainty Factors," IEEE Transactions on Human-Machine Systems, 45: 132-140, 2015.

[13] Dwivedi R, Dey S. "Score level fusion for concelable multi-biometric verification," Pattern Recognition Letters, 2018.

[14] Ghalem K.G, Hendel F. "Authentication and identification of individuals from the iris images," Second International Workshop on Mathematics and Computer Science (IWMCS), 2014.

[15] Smarandache F and Dezert J. "Applications and Advances of DSmT for Information Fusion (Collected works)," $1,2004$.

[16] Smarandache F and Dezert J. "Applications and Advances of DSmT for Information Fusion (Collected works)," $2,2004$.

[17] Smarandache F et Dezert J. "Applications and Advances of DSmT for Information Fusion (Collected works)," 3, 2009.

[18] Iris database CASIA-IrisV3, “Chinese Academy of Sciences- Institute of Automation,” Retrieved on Dec 2011. 